\title{
Observatorio
}

\section{Medios de comunicación en internet: algunas tendencias}

\author{
Por Javier Díaz-Noci
}

\begin{abstract}
Resumen: Se analizan las últimas tendencias del ciberperiodismo desde varios puntos de vista: la convergencia de medios - de redacciones, tecnológica, de contenidos y condiciones de trabajo-, la participación de los usuarios y los mitos en torno a este aspecto a veces contradichos por los datos empíricos, y finalmente se hace referencia a la polémica sobre el fin de la prensa y la influencia de internet en esa supuesta crisis. Se concluye con una reflexión sobre el cambio de los hábitos del consumo de información y la importancia de los nuevos dispositivos en todo ello.
\end{abstract}

Palabras clave: Periodismo, Internet, Ciberperiodismo, Convergencia, Tendencias.

\section{Title: Trends in internet media}

Abstract: The latest trends on online journalism are analysed in this text, from several points of view: media convergence -newsrooms, technology, contents and labour conditions-, users' participation and related myths, sometimes in contradiction with empirical evidence, and finally an analysis of the discussion about the supposed end of print media and the influence of the net in that crisis. We end up with some thoughts about the changing information consumption habits and patterns, and the importance of the new devices in it all.

Keywords: Journalism, Internet, Online journalism, Convergence, Trends.

Díaz-Noci, Javier. "Medios de comunicación en internet: algunas tendencias". El profesional de la información, 2010, noviembre-diciembre, v. 19, n. 6, pp. 561-567.

DOI: 10.3145/epi.2010.nov.01

\section{Introducción}

A MEDIADOS DE LA DÉCADA DE 1990 los primeros medios de comunicación mal que bien se pusieron en la world wide web. Se trataba de un sistema de hipertexto global más modesto que el de formulaciones teóricas anteriores (como el proyecto Xanadu de Ted Nelson), que consiguió convertirse en poco tiempo en paradigma de internet -junto con el correo electrónico, son los dos protocolos más extendidos entre los internautas-.

Y todo ello hasta el punto de popularizar en apenas una década el acceso al ciberespacio, y revolucionar a pasos no siempre tan rápidos como se anunciaba pero sí seguros e irreversibles, la edición, lectura y recuperación de información.

Los medios de comunicación y especialmente los diarios dieron a partir de mitad de esa década el salto a la Web y, aunque en muchas ocasiones han mostrado tendencias y estrategias dependientes de variables económicas y empresariales, también han innovado considerablemente el lenguaje de actualidad -y el de la ficción, pues no hemos de olvidar que una parte al menos tan importante si no más del negocio de los medios es el entretenimiento-.

Esta revolución es comparable a la que hace cinco siglos produjo en el mundo occidental la imprenta, y es imparable, si bien no ha hecho más que comenzar. Voces tan autorizadas como el informe The state of the news media del Project for excellence in journalism de la Universidad de Columbia (Nueva York, EUA) aseguran que el periodismo se encuentra en medio de una época de transformación tan trascendental como la invención del telégrafo o la televisión (Dahlgren, 2010, p. 25).

Revolución y crisis, no sólo económica y esperemos que coyuntural sino, en el caso de la industria periodística, más profunda. $\mathrm{Al}$ cierre de cabeceras -como dice Javier Guallar, probablemente 2009 haya sido en ese aspecto el peor año para la prensa- se le une "un progresivo cambio cultural -hasta ahora lento pero que 
parece acelerarse- de enorme calado" (Guallar, 2010, p. 165).

Internet se ha extendido, al menos en el mundo occidental (y hasta ahora rico) entre amplias capas de la población. Cada vez más personas, también en los países en vías de desarrollo -y en aquellos que parecen ver el final de las dictaduras comunistas, como Cuba y China-, en los cuales una gran clase media constituye la base de la democracia pero también del consumo, acceden a la Red mediante multitud de dispositivos. El ordenador de sobremesa no es el único ni el más extendido: los portátiles de diversos tamaños y capacidad y la amplia gama de teléfonos móviles de los cuales los smartphones son los más notables, están tomando el relevo. No olvidemos a la televisión como gran pantalla centralizada de información y ocio en el hogar, ya no una caja tonta sino una central multimedia.

Todo ello está provocando desde aproximadamente 1995 una profunda transformación del periodismo y en general de la forma en que los humanos nos comunicamos e intercambiamos información. Incluso los que vaticinan la muerte del periodismo (tal como lo conocíamos hasta ahora, al menos) están de acuerdo.

\section{Convergencia}

Uno de esos cambios es la convergencia de empresas, medios, tecnologías y lenguajes. "Convergencia" es un término muy de moda desde que en la década de 1980 Ithiel De-Sola-Pool lo aplicó al mundo de la comunicación, si bien no siempre se define de forma unívoca. Para Henry Jenkins, es el espacio "donde los antiguos y los nuevos medios chocan, donde las bases y los medios corporativos se entrecruzan, donde el poder de los productores de medios y el poder de los consumidores de medios interactúan de manera impredecible" (Jenkins, 2006).

Para nosotros, que usamos la definición propuesta por Ramón Salaverría, Pere Masip y José-Alberto García-Avilés en Convergencia digital. Reconfiguración de los medios de comunicación en España, se trata de "un proceso multidimensional que, facilitado por la implantación generalizada de las tecnologías digitales de telecomunicación, afecta al ámbito tecnológico, empresarial, profesional y editorial de los medios de comunicación, propiciando una integración de herramientas, espacios, métodos de trabajo y lenguajes anteriormente disgregados, de forma que los periodistas elaboran contenidos que se distribuyen desde múltiples plataformas, mediante los lenguajes propios de cada una" (Salaverría; García-Avilés; Masip, 2010, p. 48).

La convergencia ha afectado a diversos aspectos de la comunicación. Desde una perspectiva técnica supone una mayor portabilidad de los datos, la existencia de redes descentralizadas, una abundancia de tecnología y aplicaciones, el acercamiento de los procesos de creación y producción, la posibilidad de que los usuarios se conviertan en productores, y la aceleración del tiempo y los límites temporales.

Desde un punta de vista organizativo se detecta una tendencia en los medios hacia la producción integrada, los profesionales multitarea, la distribución multiplataforma y la audiencia activa. En un primer momento es probablemente la distribución para diversas plataformas la que obtiene más éxito. De hecho, a la distribución en papel -y en formato audiovisual cuando se trata de medios matrices de este tipo-, se añaden la edición web, para PDAs, teléfonos móviles e iPhones, y ahora ediciones para Kindle u otros formatos de libro electrónico (si bien tímidamente) y para $i P a d$, a partir de su comercialización en 2010.

\section{"La convergencia propicia la integración de herramientas, espacios, métodos de trabajo y lenguajes anteriormente disgregados"}

En teoría las empresas mejor preparadas serían aquellas que ya tienen redacciones integradas y personal polivalente, empresas que funcionan como grupos y además funcionan con la lógica 7 x 24 (siete días a la semana durante 24 horas), cuyos profundos cambios han sido estudiados por Rosenberg y Feldman, (2008), entre otros. Por el contrario, la realidad, al menos entre nosotros, no es tan idílica. A partir del estudio llevado a cabo por un grupo de 25 investigadores $^{1}$, al menos en España podemos afirmar que la mayoría de las empresas se encontraban en procesos en fase inicial o intermedia, en los que predominan las redacciones que se encuentran en un mismo espacio, que comparten algún tipo de contenidos y muestran una mínima coordinación organizativa común. Son también abundantes las redacciones separadas que comparten contenidos pero que funcionan mediante estructuras organizativas separadas.

A la vez no podemos olvidar, aunque aquí no queden sino apuntados, otros efectos colaterales. La compensación es uno de ellos. Una mayor integración organizativa facilita y posibilita la existencia de condiciones de trabajo comunes. Sin embargo la mayoría de los grupos mediáticos españoles disponen de empresas diferentes para cada medio y de convenios específicos para cada uno de ellos. Por regla general la peor situación en cuanto a condiciones de trabajo la sufren los redactores de los cibermedios, que ganan menos dinero 
y tienen peores horarios y turnos de trabajo que los colegas de prensa impresa.

Se cumple así la predicción que en 2005 hacía Steve Klinenberg, para quien la combinación de posibilidades tecnológicas y las presiones económicas para llevar a cabo procesos de convergencia en los medios lleva aparejada un empeoramiento de las condiciones laborales de los trabajadores, al menos en cuanto a tiempo, porque era una queja generalizada la dificultad creciente para preparar y escribir buenos reportajes (Klinenberg, 2005, p. 62). Se trata de un reto también

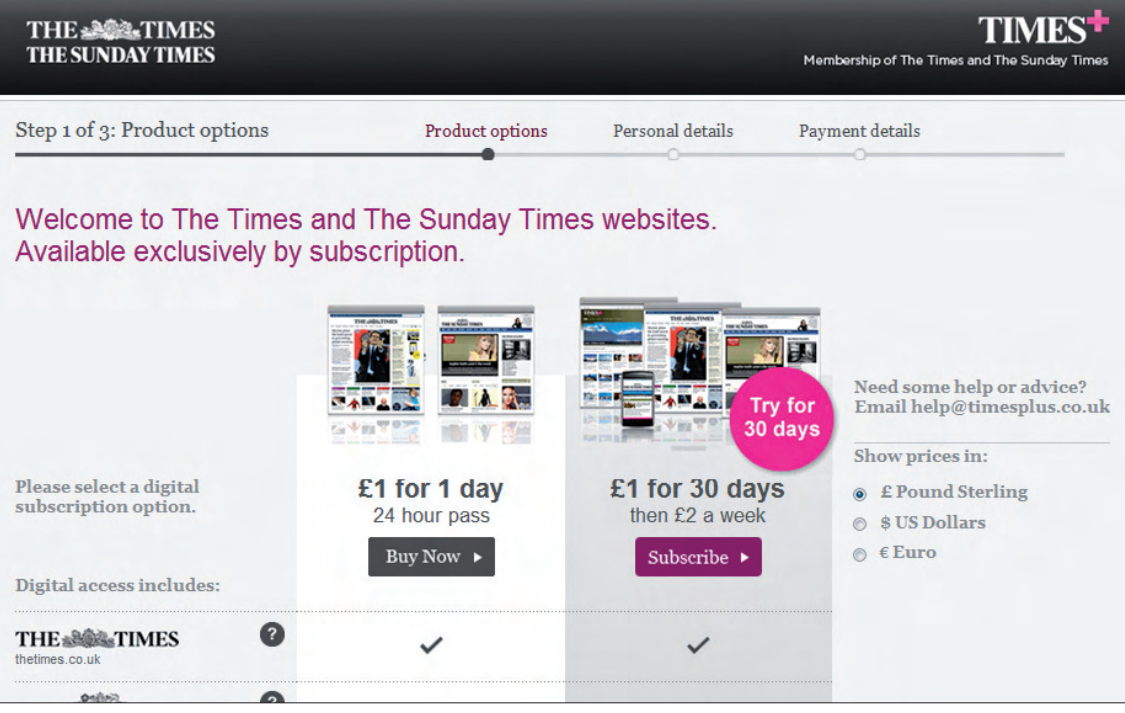

Acceso de pago a The Times, http://www.timesplus.co.uk puesto de manifiesto por Gracia Lawson-Borders (2006, p. 16), otro de los nombres de referencia en el estudio de la convergencia mediática.

\section{Participación del usuario}

Otros fenómenos son también manifiestos, aunque deben ser contemplados en su verdadera dimensión, sin caer en el determinismo o en el optimismo no justificado. Uno de ellos es la incorporación de los usuarios como autores: el periodismo participativo, en sus diferentes grados de elaboración. Hasta Rupert Murdoch (en su discurso a la American Association of Newspapers Editors en abril de 2005) vio la importancia del papel del usuario: "Lo que está sucediendo es una revolución en la manera cómo los jóvenes acceden a sus noticias. La próxima generación tiene diferentes expectativas sobre el tipo de noticias que recibirán, incluyendo dónde, cuándo, cómo y de quién las van a obtener". La sentencia de Murdoch es que "los diarios sólo pueden sobrevivir con estrategias multimedia”.

\section{"Hay que destacar la incorporación de los usuarios como autores: el periodismo participativo"}

Por lo que se refiere a la dimensión participativa de los usuarios y a su potencial democrático todo parece indicar, como decía el empresario Martín Varsavsky en una entrevista a la edición española de la revista $E s-$ quire en mayo de 2009, que "el que escribe en internet cuida lo que publica porque sabe que la respuesta va a ser inmediata. Los comentarios de los lectores obligan al periodista a ser mejor, a ser más veraz y los medios online están más cerca de llegar a la objetividad de los de papel".
Sin embargo la realidad muestra un panorama bastante menos alentador. Es cierto que la web 2.0 teóricamente concede un nuevo protagonismo a la conversación social. Pero es más cuestionable por ejemplo que el estado actual de los comentarios de los medios digitales, especialmente el de aquellas cabeceras que por provenir de diarios impresos de prestigio serían de referencia, nos dibuje una mayor calidad del debate. La calidad de los argumentos y de las deliberaciones es importante; que se haya aprovechado o no el potencial de los nuevos medios es algo que discuten obras recientes (Jones, 2009; Dahlgren, 2009). Desde un punto de vista económico, en esta denominada "economía de la atención" los medios digitales, sobre todo los diarios, ofrecen como valor añadido mecanismos de participación (y de fidelización).

A la vez las audiencias son cada vez más fragmentadas y huidizas, buscan estímulos inmediatos y satisfacciones igualmente celéricas, como ha puesto de manifiesto Sonia Livingston (1999).

\section{EI anunciado fin de la prensa}

Curiosamente ha sido Murdoch, que ha comprado The Wall Street journal y lo ha convertido en un diario de información general -ya no sólo económica- con el propósito declarado de competir con el New York times, quien ha anunciado su intención de extender el modelo de pago a toda la prensa digital. En julio de 2010 Rupert Murdoch avanza su estrategia de volver a un modelo de pago por suscripción con Times online. El argumento: el lector de calidad estará dispuesto a pagar por la información de calidad. No olvidemos que el opuesto al serio y adusto Times, el popular tabloid The sun, también es de Rupert Murdoch.

Otros indicios apuntan en dirección contraria. El 11 de septiembre de 2009 Elpaís.com pregunta a sus lectores/internautas si estarían dispuestos a pagar por 
"contenidos de calidad". El 14 de septiembre de 2009 la encuesta indica que sólo el $12 \%$ de quienes han respondido estarían dispuestos a rascarse el bolsillo por esos contenidos. El 86\% responde con un rotundo "no" a la pregunta.

La industria periodística se ha dedicado durante 2009 y 2010 a debatir si estamos ante el fin del periodismo, o tan sólo ante el fin de los diarios tal y como hasta ahora los conocíamos. Según un estudio de Martin Langeveld para el Nieman Journalism Lab, recogido por Anthony Smith, "los lectores pasan cuatro veces más tiempo leyendo

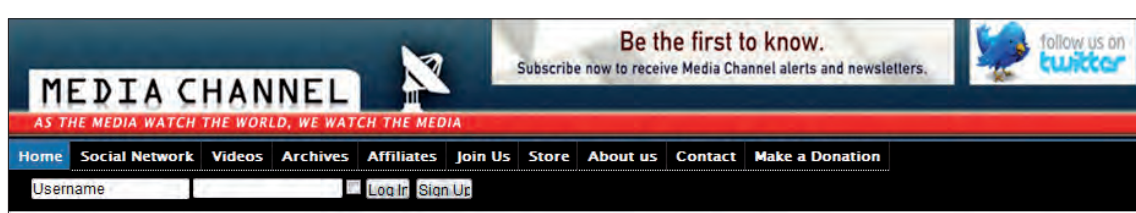
las copias impresas que las versiones electrónicas", y de hecho "los periódicos impresos captan a una audiencia seis veces mayor que la de la versión electrónica" (Smith, 2010, p. 14). Estos datos desmentirían la visión apocalíptica que los propios diarios dan de la supuesta crisis provocada por los medios digitales.

$\mathrm{Y}$ es que los grandes diarios no siempre han acertado a formar comunidades -de lectores y de anunciantes- en internet, cosa que sí consiguieron en el siglo $\mathrm{XX}$ con el soporte impreso.

La formación y cohesión de comunidades de intereses han sido desarrolladas más bien por iniciativas como MediaChannel, One world o Corporate watch, y pequeños diarios, como el estadounidense Bakersfield. com, como en su día lo hizo Nando.net. Junto con los grandes nombres procedentes del periodismo pre-internet, se han llevado a cabo otras formas de comunicación, desde aquellas que imitan a la prensa -y a la más ideologizada y de derechas, como Politico.com o el Huffington Post- hasta las que confían, como el medio español La información.com, en una mezcla de informaciones propias redactadas por sus periodistas e informaciones ajenas ofrecidas al modo de los agregadores y jerarquizadas según algoritmos complejos.

Otras iniciativas combinan el periodismo ciudadano y el profesional, como OhMyNews o el francés Rue89, que se deciden por medios nativos digitales, como el catalán Vilaweb.cat o el checo NaseAdreza. $c z$, y tampoco conviene perder de vista la decisión de Guardian unlimited de confiar las noticias galesas a bloggers locales. Conviven junto a medios generalistas, medios espe-

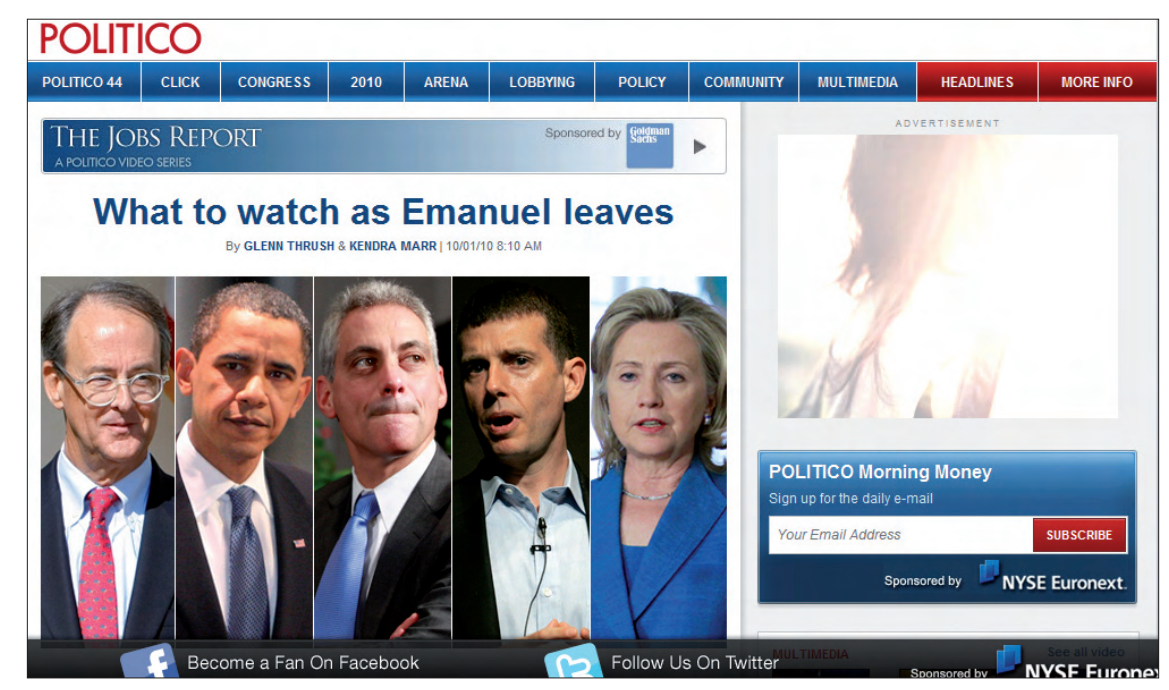

Politico.com, http://www.politico.com cializados de referencia -y edición exclusivamente digital- como Technorati o Gizmodo, iniciativas independientes para financiar grandes reportajes con los temas que interesan a los ciudadanos, como el Independent media center, PrePublica, Chicago news cooperative o NewAssignment.net ("An experiment on open-sourced reporting"), informaciones alternativas como la de Canalsolidario.org o Periodismo humano, o creadas por redactores del mundo impreso en tiempos de crisis, como el español Cuarto poder.

\section{"Las tabletas permiten acceder a la información en cualquier momento y lugar con una buena definición"}

Sin olvidar un ejemplo acerca de cómo el compromiso de una periodista, Judith Torrea, ofrece al mundo 


\section{CIUDAD JUÁREZ, EN LA SOMBRA} DEL NARCOTRÁFICO

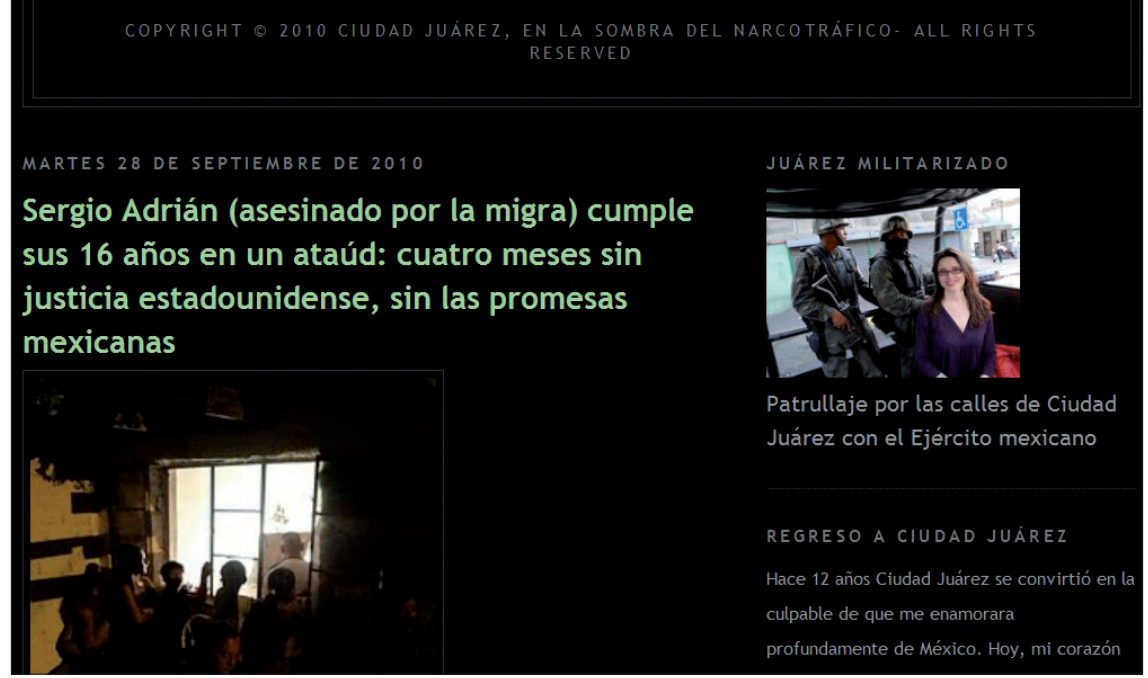

Ciudad Juárez en la sombra del narcotráfico, http://juarezenlasombra.blogspot.com/ recurso que está empezando a ser ubicuo y gratuito.

Podríamos estar cerca de un nuevo salto cualitativo en la manera en que nos relacionamos con la información digital. Basta fijarse por ejemplo en el consumo de música, que en gran medida ha abandonado la dependencia del soporte físico (empresas como Spotify se han dado cuenta: la gente no quiere tener muchos $\mathrm{CD}$, quiere tener acceso a muchas canciones), o en el consumo de series de televisión: buena parte se visualiza no de forma síncrona a su emisión sino mediante los archivos disponibles en internet.

La cultura de la propiedad $-y$ de la necesidad de que la informala mejor información posible sobre una zona y un tema de alto riesgo: el narcotráfico en la mexicana Ciudad Juárez, y lo hace empleando una plataforma de blog pública: Ciudad Juárez en la sombra del narcotráfico.

\section{http://juarezenlasombra.blogspot.com/}

También hay organizaciones universitarias que velan por la exactitud de los hechos que expresan los políticos estadounidenses en los medios (FactCheck.org) o iniciativas que desmienten informaciones inexactas en internet, como Snopes.com (Meyer, 2010, p. 44).

A pesar de estas evidencias, la opinión difundida por los propios medios se debate entre la reivindicación de los valores del periodismo -si bien es curioso que quien los reclame sean empresarios como Rupert Murdoch, conocido por sus prácticas monopolísticasy su contribución a la democracia y la búsqueda de tablas de salvación. O más bien de tabletas.

\section{¿Cambio en el acceso y consumo de información?}

Las tabletas planas, cuya idea y prototipos ya existían desde los años 90, se han hecho realidad (o sea, se han comercializado) en 2010.

El iPad de Apple se ha situado en el mercado de manera decidida, aunque otras similares se van anunciando en una competencia, similar a la de los clónicos del pc de IBM en 1980-1990, que se adivina reñida. Las tabletas permiten acceder a la información en cualquier momento y lugar, con una buena definición, la posibilidad de llevar cargada una biblioteca de medios (música, fotos, vídeos, información) y sobre todo disponer de multitud de aplicaciones baratas que hacen de todo. Y por supuesto con conexión wi-fi, un ción llegue en un soporte físico- ha dado paso a la cultura del acceso. A este mercado se orientan las nuevas tabletas tipo iPad. Y si bien es el consumo de ficción televisiva el que crece y lleva camino entre las nuevas generaciones de convertirse en una forma de recepción individual $^{2}$ como hoy es la del libro o los periódicos, han sido empresas como New York times o revistas como Wired las que han visto en este nuevo aparato la salvación para sus productos.

\section{"La cultura de la propiedad ha dado paso a la cultura del acceso"}

Los directivos de Wired, revista de tendencias del mundo digital publicada por Condé-Nast, cuya versión impresa ha pasado por serias dificultades en diversos momentos de su existencia, asegura que el producto en el que confían no es su versión web -que sí les reporta dividendos-, sino que lo que siempre han querido hacer es una revista impresa en formato digital. Así que han lanzado una app para iPad. Utilizando el programa Adobe Air -curiosamente, parece que es el software más extendido en estos primeros momentos para producir revistas para $i P a d$, cuando Apple y Adobe mantienen una guerra abierta a causa del Flash-Wired ha sido una de las primeras publicaciones en apostar por una edición para la tableta. Se trata de una revista impresa en formato digital con realidades aumentadas, básicamente.

Como ya ha demostrado Pere Masip (2010), aunque se emplea cada vez más contenido multimedia, por 


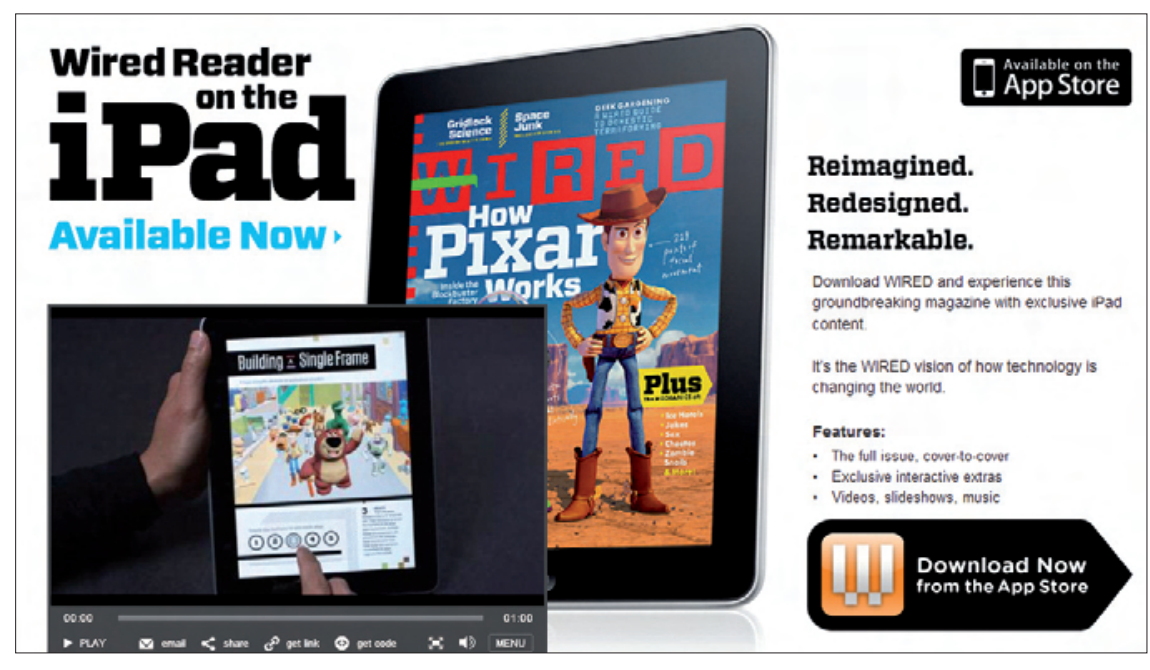

Versión de Wired para iPad, http://www.wired.com/magazine/ipad

foto de una noticia sea, a la orden de un clic, un vídeo. Multimedia e interactivo, así se definía en 19951996 el entonces llamado periodismo electrónico. Y exactamente así se define el futuro de estas nuevas tabletas. No porque sea una realidad sino porque, quince años después, ése es precisamente el gran reto: el desarrollo de la hipertextualidad, la multimedialidad -no meramente yuxtapuesta, sino integrada- y la interactividad.

Hoy empresas como la de Time sports illustrated siguen imaginando y elucubrando; ahí están el Man-

ejemplo en forma de vídeo, de momento hay "algunas certezas y muchas dudas". Según el estudio de Masip, el lenguaje de los vídeos informativos está más cerca en los cibermedios del entretenimiento que de la información. Piezas muy cortas, de 40 segundos, con un lenguaje sencillo y dinámico.

\section{"El gran reto es la integración de la hipertextualidad y la multimedialidad, y la interactividad"}

No queda claro en cambio hasta qué punto estas ediciones para tableta ofrecerán voz a los lectores en la misma medida que las ediciones web, o bien los lectores tendrán un producto más volcado al multimedia, pero muy basado en el diseño impreso. Es posible que estemos asistiendo a una especialización de las ediciones: la de la Web sería la más popular, mientras que los servicios premium se encontrarían en las ediciones para tableta. Hay algunas iniciativas en forma de aplicaciones para iPad o iPhone que nos dan idea de esta tendencia a mimetizar el diseño gráfico, una de las grandes carencias de la world wide web. Flipboard permite al usuario confeccionarse una revista a medida con noticias extraídas de las redes sociales. En esa edición personalizada en la línea de lo estudiado por Knobel y Lankshear (2010), lo que ellos llaman DIY (do it yourself), sólo se ofrece el inicio de la noticia y la información depende de un hipervínculo que enlaza con la fuente original, lo que salvaguarda los derechos de autor. La flexibilidad de edición y el apoyo en las redes sociales, características de la web 2.0, podrían apuntar una tendencia de futuro.

Algunas de las fantasías que Roger Fidler predicaba en 1994 se han hecho realidad: por ejemplo, que la hattan project, Tablet magazine. Ni siquiera el New York times descarta, quizá allá por 2015, convertirse en un medio exclusivamente digital, como ya ha hecho (por razones económicas, todo hay que decirlo) el que fue el primer diario brasileño en ofrecer una edición digital, el Jornal do Brasil, en agosto de 2010.

\section{"Las tabletas no salvarán el periodismo (impreso), pero potenciarán el audiovisual"}

Podríamos estar frente a un producto claramente sustitutivo, al contrario que la Web (aunque el discurso de la década de 1990 y buena parte de la primera del 2000 ha sido por parte de los editores de prensa plantear si la Web mataría la imprenta). Pablo Boczkowski (2009) afirma que el aumento de la transparencia y del número de canales trae como consecuencia una intensificación del mimetismo, es decir, lo que tenemos es más lugares donde elegir contenidos, y ahora incluso diseño, similares.

Lo cierto es que si se extiende el uso de las tabletas hasta convertirse en ubicuas, y el acceso a internet se considera un derecho de todo ciudadano como ya sucede en Finlandia, las empresas se ahorrarían los gastos en impresión y distribución física de ejemplares, paradigma de la edición impresa.

Estas pantallas podrían estar también cambiando la forma de consumo del audiovisual que hasta ahora predominaba: la comunitaria - el cine- o familiar -la televisión- para dar paso a un acceso similar al del libro: individual y en silencio. La televisión no está interesada en un dispositivo que imita el papel, sino en una pantalla portátil que permita reproducir sus contenidos, por lo que es muy posible que los dispositivos e-paper tengan 
una vida efímera o se conviertan en un mero instrumento auxiliar siempre que su precio sea muy barato.

Muy probablemente, como dice un artículo de Jack Shaffer (2009), las tabletas no salvarán el periodismo (impreso). Potenciarán en cambio el audiovisual, cuyo lenguaje estaría en situación ventajosa frente a la mera palabra.

\section{Notas}

1. Resultado del proyecto coordinado de $\mathrm{I}+\mathrm{D}$ Convergencia digital en los medios de comunicación, referencias SEJ2006-14828-C06-01, -02, -03 y -04, financiado por el Ministerio de Ciencia e Innovación de España. Se detiene al inicio de la crisis económica, lo cual es otro factor a tener en cuenta, porque detiene, cuestiona o congela muchos procesos de convergencia.

2. Sólo a manera de ejemplo, en el curso 2010-2011 se prevé que el $80 \%$ de los colegios públicos de Barcelona dispondrá de forma exclusiva de libros de texto digitales, y no impresos en papel. Esta generación, por tanto, podría no ser educada ni estar familiarizada en absoluto con el consumo de literatura impresa.

\section{Bibliografía citada}

Boczkowski, Pablo. "Materiality and mimicry in the journalism field". En: Zelizer, Barbie (ed.). The changing faces of journalism. London: Routledge, 2009 , p. 56-67.

Dahlgren, Peter. "El horizonte de la democracia". Infoamérica. Iberoamerican communication review. Málaga: Cátedra Unesco de la Universidad de Málaga, 2010, n. 2, p. 21-37.

http://www.infoamerica.org/icr/num02/dahlgren.pdf

Dahlgren, Peter. Media and political engagement: citizens, communication and democracy. New York: Cambridge University Press, 2009.

Dailey, Larry; Demo, Lori; Spillman, Mary. "The convergence continuum: a model for studying collaboration between media newsrooms". Atlantic journal of communication, 2005, v. 13, n. 3, p. 150-168.

Guallar, Javier. "Prensa digital en 2009". Anuario ThinkEPI, 2010, v. 4 pp. $165-173$.

http://eprints.rclis.org/18874/1/anuariothinkepi10_guallar_prensa_ digital_2009.pdf

Jenkins, Henry. Convergence culture. Where old and new media collide. New York: New York University Press, 2006.
Jenkins, Henry. "The cultural logics of media convergence". International journal of cultural studies, 2004, v. 7, n. 1, p. 33-43.

Jones, Alex. Losing the news: the future of news that feeds democracy. New York: Oxford University Press, 2009.

Klinenberg, Steve. "Convergence: news production in a digital age". The annals of the American Academy of Political and Social Science, 2005, v. 597, n. 1 , p. $48-64$

Knobel, Michele; Lankshear, Colin (eds.). DIY media: creating, sharing and learning with new technologies. New York: Peter Lang, 2010.

Lawson-Borders, Gracie. Media organizations and convergence. Case studies of media convergence pioneers. New Jersey: Lawrence Erlbaum, 2006.

Livingston, Sonia. "New media, new audiences?" New media and society, 1999 , v. 1, n. 1, p. 59-66.

López, Xosé; Pereira, Xosé (eds.). Convergencia digital. Reconfiguración de los medios de comunicación en España. Santiago de Compostela: Universidade de Santiago, 2010

Masip, Pere. "Vídeo en los cibermedios. Algunas certezas y muchas dudas". Anuario ThinkEPI, 2010, v. 4, pp. 180-185.

Meyer, Philip. "La supervivencia en la web 3.0". Infoamérica. Iberoamerican communication review. Málaga: Cátedra Unesco de la Universidad de Málaga, 2010, n. 2, p. 39-57.

http://www.infoamerica.org/icr/num02/meyer.pdf

Rosenberg, Howard; Feldman, Charles S. No time to think: The menace of media speed and the 24-hour news cycle. New York: Continuum, 2008.

Salaverría, Ramón; García-Avilés, José A.; Masip, Pere. "Concepto de convergencia periodística”. En: López-García, Xosé; Pereira-Fariña, Xosé (coords.). Convergencia digital. Reconfiguración de los medios de comunicación en España. Santiago de Compostela: Servicio Editorial de la Universidad de Santiago de Compostela, 2010, pp. 41-63.

Shaffer, Jack. "The tablet hype". Slate, 22 December 2009. http://www.slate.com/id/2239557/

Smith, Anthony. "El periódico que viene. Reconstrucción del escenario mediático". Infoamérica. Iberoamerican communication review. Málaga: Cátedra Unesco de la Universidad de Málaga, 2010, n. 2, p. 7-19.

http://www.infoamerica.org/icr/num02/smith.pdf

Javier Díaz-Noci. Universitat Pompeu Fabra, Barcelona. javier.diaz@upf.edu

\section{Próximos temas centrales}

Enero 2011

Marzo 2011

Mayo 2011

Julio 2011

Septiembre 2011

Noviembre 2011
Psicología y sociología de la información

Archivos administrativos e intranets

Información de las administraciones públicas

Fotografía y bancos de imágenes

Inteligencia competitiva

Fuentes de información 2.0

Los interesados pueden remitir notas, artículos, propuestas, publicidad, comentarios, etc., sobre estos temas a: http://recyt.fecyt.es/index.php/EPI/index 